\title{
Structural, Micro-structural and Electronic Structure Evolution in Polycrystalline Perovskite Electro-ceramics Based on $\mathrm{Ba}_{1-\mathrm{x}} \mathrm{Ca}_{\mathbf{x}} \mathrm{Ti}_{0.9} \mathrm{Zr}_{0.1} \mathrm{O}_{3}$
}

G. Herrera-Pérez ${ }^{1}$, A. Reyes-Montero ${ }^{2}$, K. Campos-Venegas ${ }^{1}$, R. Gómez-Bartres ${ }^{1}$, J. Canche-Tello ${ }^{3}$, M. $^{2}$ E. Montero-Cabrera ${ }^{3}$, J. R. Plaisier ${ }^{4}$, D. Eichert ${ }^{4}$, A. Reyes-Rojas ${ }^{1}$ and L. E. Fuentes-Cobas ${ }^{1}$

1. Depto. de Física de Materiales, Centro de Investigaciones en Materiales Avanzados S. C., Chihuahua, Chih., Mexico.

2. Instituto de Investigaciones en Materiales, Universidad Nacional Autónoma de México, Mexico City, Mexico.

3. Depto. de Medio Ambiente y Energía, Centro de Investigaciones en Materiales Avanzados (CIMAV) S. C., Chihuahua, Chih., Mexico.

4. Elettra-Sincrotrone Trieste S.C.p.A, Basovizza, Trieste, Italy.

The nature of the piezo/ferro-response of lead-free electro-ceramics (bulk [1] or thin films [2,3]) based on $\mathrm{Ba}_{1-\mathrm{x}} \mathrm{Ca}_{\mathrm{x}} \mathrm{Ti}_{0.9} \mathrm{Zr}_{0.1} \mathrm{O}_{3}$ (denoted in this work as $\mathrm{BCZT}$ ) stoichiometry has been the attraction of the scientific community. Several reports about the structural and electronic structure provides information about their non-centrosymmetric structure and the important contribution of the covalent character presented in this ionic composition [1]. However, during the synthesis of these compounds by soft chemistry routes such as sol-gel; texture (the preferred crystal orientation) could favorer and the morphology of samples would be affected. The purpose of this work was to monitor the evolution of texture and the morphology in BCZT compounds (at different concentrations of $\mathrm{Ca}, \mathrm{x}=0,0.1,0.125$, $0.15,0.175,0.2)$ by high resolution X-ray diffraction (HR-XRD), scanning electron microscopy/energydispersive X-ray spectroscopy (SEM-EDS) and micro X-ray absorption near edge spectroscopy ( $\mu$ XANES).

The samples were prepared by the modified Pechini method [1] and they were sintered at $1400^{\circ} \mathrm{C}$ for 5 h. HR-XRD and $\mu$-XANES experiments took place at the 7.1 MCX and the 10.1L X-ray fluorescence beamlines at the Elettra Sincrotrone Trieste (EST). The surface morphology, elemental microanalysis and mapping were recorder using SEM-Hitachi SU3500 with a EDS AZtecEnergy acquisition software with X-Max ${ }^{\mathrm{N}}$, Oxford Instruments.

Figure 1 (a-c) shown three representative HR-XRD patterns of BCZT with narrow peaks that suggest that grains are in the micron scale. It can be also observed the presence of texture (panel $b$ and $c$ ) in comparison with the reference $\mathrm{BaTiO}_{3}$ compound (panel a). The HR-XRD patterns were refined by the Rietveld method using Fullprof version 2017 and the results suggest a perovskite structure with a single tetragonal phase (P4mm space group). The comparison between SEM micrographs reveals a nonhomogeneous rod polyhedral-type shapes and faceted quasi-cubs as it can be seen in Figure 1d. Panel e shows a duplex morphology consisting of small grains and abnormally rod-large grains. Faceted quasihexagons grains with the presence of terraces is shown in Figure 2a. These microstructural results suggest an abnormal grain growth during the sintering process. Furthermore, elemental analysis (Figure 2b) was carried out to calculate elemental composition and their distribution throughout the grains. For example, the molecular formula proposed as $\mathrm{Ba}_{0.9} \mathrm{Ca}_{0.1} \mathrm{Ti}_{0.9} \mathrm{Zr}_{0.1} \mathrm{O}_{3}$ is consistent with the estimated atomic percentages by this characterization. Elemental mapping was carried out in sintered BCZT observing that all elements (barium, calcium, zirconium, titanium and oxygen) are homogeneously distributed throughout BCZT. The experimental absorption spectra of BCZT (Ti K-edge) shown three main features in the pre-edge region (4965-4985 eV). The first one at $4969 \mathrm{eV}$ corresponds to a dipole- 
forbidden transition of a $1 \mathrm{~s}$ electron into the $t_{2 \mathrm{~g}}$ states for octahedrally coordinated Ti. The dipoleallowed transition of a 1s electron into hybridized 3d-4p states with $e_{g}$ symmetry is located at $4972 \mathrm{eV}$. The last one corresponds to the transition of a $1 \mathrm{~s}$ electron into the unoccupied $3 \mathrm{~d}$ states of neighboring Ti cations (4976 eV). The intensity of features labeled in $4969 \mathrm{eV}, 4972 \mathrm{eV}$ and $4976 \mathrm{eV}$ decreased in this region, when $\mathrm{Ca}$ concentration was increasing [4].

\section{References:}

[1] G. Herrera-Pérez et al, J. Appl. Phys. 120 (2016), p 094303.

[2] G. Herrera-Pérez et al, Microsc. Microanal. 23 (Suppl. 1) (2017), p. 1648.

[3] G. Herrera-Pérez et al, Mat. Res., 2018 doi: 10.1590/1980-5373-mr-2017-0605.

[4] G. Herrera-Pérez acknowledge funding from the Basic Research Project CONACyT-SEP Grant No. 253605, Cátedra CONACyT Grant No. 2563 and CONACyT-SNI 1. Synchrotron experiments were performed under proposals EST No. 20165097 and No. 20165205.
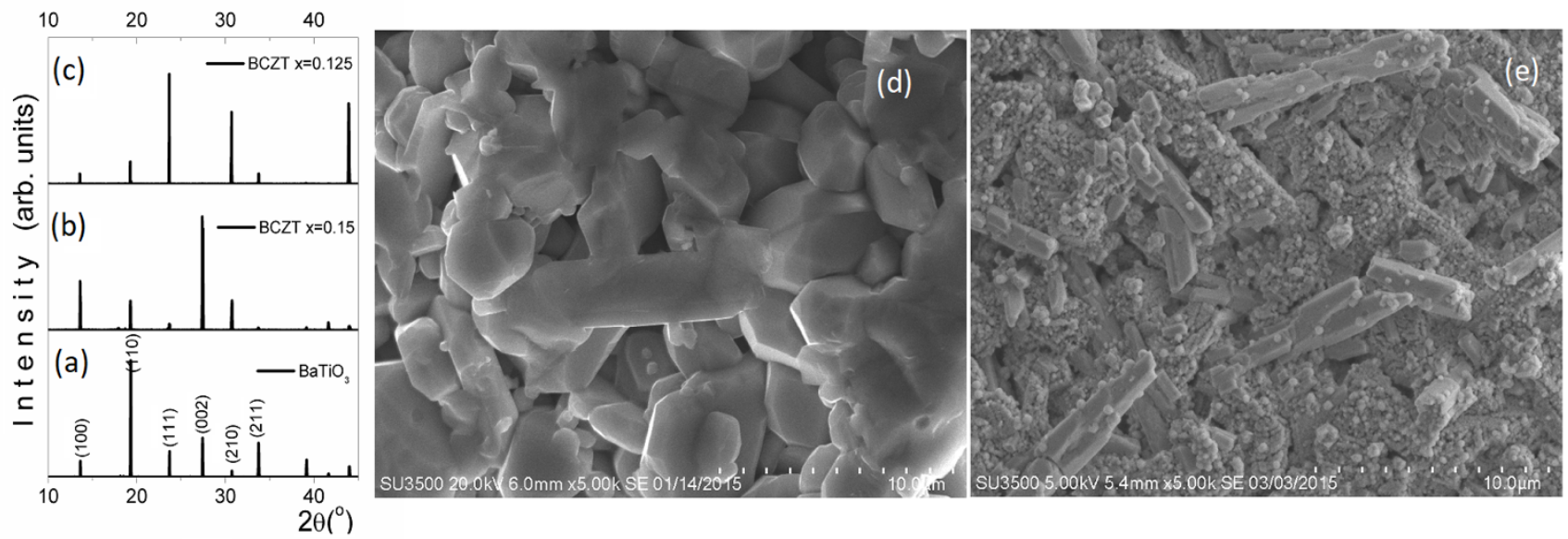

Figure 1. HR-XRD patterns for the: (a) reference compound $\mathrm{BaTiO}_{3}$, (b) $\mathrm{BCZT}$ with $\mathrm{x}=0.15$, (c) $\mathrm{BCZT}$ with $\mathrm{x}=0.125$. SEM micrographs for: (d) BCZT with $\mathrm{x}=0.1$ (e) BCZT with $\mathrm{x}=0.15$.
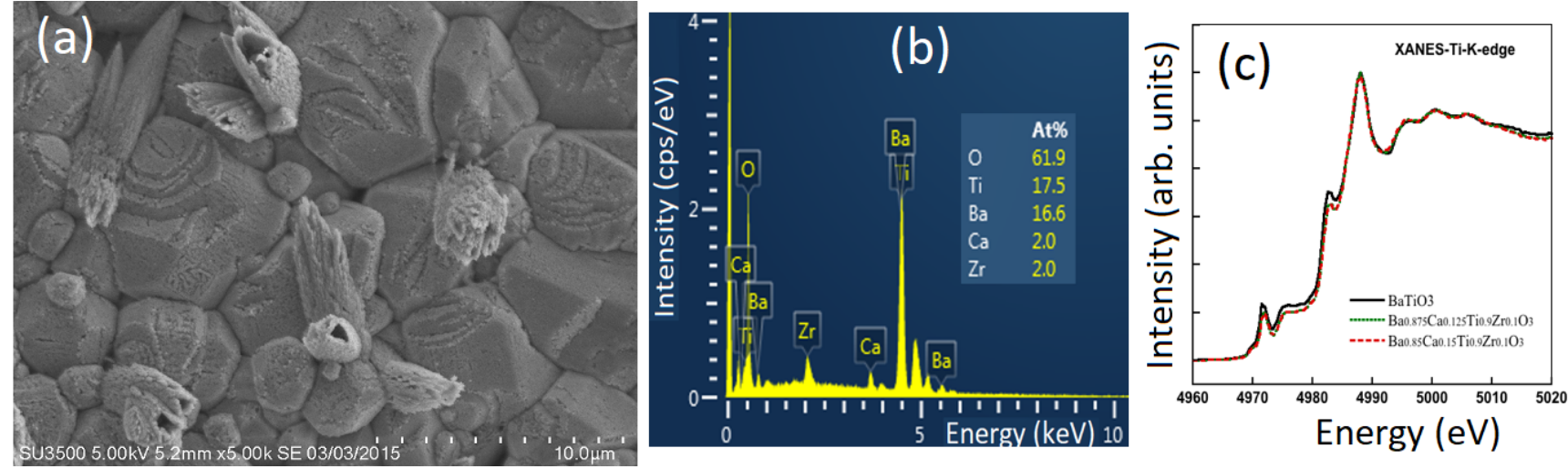

Figure 2. (a) SEM micrograph for the BCZT with $\mathrm{x}=0.125$. (b) SEM/EDS, elemental microanalysis for the $\mathrm{Ba}_{0.9} \mathrm{Ca}_{0.1} \mathrm{Ti}_{0.9} \mathrm{Zr}_{0.1} \mathrm{O}_{3}$ compound. (c) Comparison between $\mu$-XANES for three different of BCZT compositions. 